\title{
PREVENTION AND TREATMENT OF NEURO-SYPHILIS.
}

\author{
By B. BUCKLEY SHARP, M.D., M.R.C.P. \\ (Physician to the Princess Beatrice and Evelina Hospitals; \\ Assistant to the Neurological and Venereal Disease Depts., Royal Northern Hospital; \\ Assistant in the Venereal Disease Dept., St. Bartholomew's Hospital.)
}

\section{A.-Prevention.}

This depends on (I) The thorough and intensive treatment of early syphilis. (2) The routine examination of the cerebro-spinal fluid to discover those cases in which there are persistent changes despite such adequate early treatment. Special treatment of those cases in which such changes are present.

The cerebro-spinal fluid must be examined in all cases whether the blood Wassermann has or has not been positive, and whether or not it has become persistently negative under treatment in the cases initially positive. The chance of an irreversibly positive cerebro-spinal fluid is twice as great when treatment is begun in early secondary syphilis (I.3 per cent.), as when it is begun in the seronegative primary stage (0.6 per cent.-Stokes I934). Should the blood remain positive despite a full amount of standard treatment, a negative cerebro-spinal fluid after four years is strong evidence that it will never become positive. Cerebrospinal fluid examination must not be omitted in old-standing, latent or manifest somatic syphilis coming under treatment for the first time. Lomholt believes that the cerebro-spinal fluid may remain negative during standard treatment, becoming abnormal only when treatment is stopped. Therefore he advises that the fluid be re-examined one year after cessation of treatment. Burke advises that the fluid be examined at the end of routine treatment, and twice during the two years of observation.

There is no evidence that thorough treatment of syphilis by arsenical drugs predisposes to an increased incidence of neuro-syphilis-the evidence is to the contrary and has been confirmed by a European survey made in I928 by the German Dermatological Society. The incidence of tabes and dementia paralytica has been lowered since the introduction of more effective chemo-therapeutic weapons in the last 25 years. But it cannot be too forcibly stressed that when the cerebrospinal fluid remains abnormal, ordinary standard treatment is not effective in preventing parenchymatous neuro-syphilis. There is some evidence that in such circumstances the incubation period of parenchymatous neuro-syphilis, as exemplified by dementia paralytica, may be shorter in cases that have had adequate standard treatment than in completely untreated or inadequately treated cases. (Nicol \& Hutton 1937). The longest incubation period encountered in treated women was 20 years (average I4 years), and in treated men 22 years (average I7 years); the longest observed incubation period in women, never treated with arsenic or bismuth, was $3 \mathrm{I}$ years, and in men 50 years. In Nicol and Hutton's series the incubation period in women tended to be shorter than in men.

All authorities are agreed, I think, that inadequate or irregular early treatment increases the risk of neuro-syphilis developing later. It is probably more dangerous from this point of view than no treatment at all, and inhibits the development of active immunity without eradicating the infection. Burke maintains that a rest period or default from treatment for three consecutive weeks during the first three months is most dangerous. 
It is outside the scope of this paper to set out the technique and details of standard treatment of syphilis or to discuss the relative merits of continuous treatment with alternating courses of trivalent arsphenamine (arsenobenzol), and bismuth preparations and of intermittent treatment composed of courses of arsphenamine and bismuth administered concurrently with rest periods. It is assumed that the reader is familiar with this aspect of the subject. It appears likely from the study of family histories that there may be a predisposition in some persons to an attack upon the central nervous system when syphilis is contracted. There are also suggestive histories on record indicating the existence of a specially neurotropic strain of the treponema pallidum.

In the prevention of clinical neuro-syphilis when latent neuro-syphilis is discovered by routine lumbar puncture after two years or more of adequate standard treatment, the same methods must be employed as in the treatment of clinical neuro-syphilis (see later). It may be noted here that malaria in primary syphilis cannot be relied upon to prevent the development of secondary syphilis and that its use during the first two years of treatment is not justified.

The technique of lumbar puncture:-The goal to be aimed at is the avoidance of post-lumbar puncture headache and vertigo, which may persist in some cases up to two weeks when the patient assumes the erect position, the symptoms disappearing when the patient lies down. This indicates that the cause is a persistent lowering of the cerebro-spinal fluid pressure, which can only be explained by the continuing leakage of fluid from the puncture hole in the membranes. It is impossible to predict which patients will be affected and which not, though neuro-syphilitics are less likely to suffer than the neurologically healthy. The solution of the problem is to pierce the membranes with a very fine needle, but a sufficiently fine ordinary needle is not rigid enough. Dattner overcame the difficulty by designing a composite needle with an outer and an inner tube, the latter being very fine, longer than the former in which it fits, and capable of being withdrawn within the outer tube and projected at will half an inch beyond it. The inner tube is fixed in either position by a screw at the butt (Harrison's modifica-

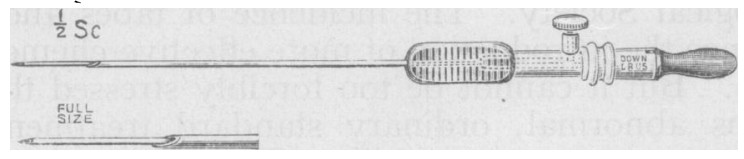

tion). In use the outer needle pierces the skin and tissues but is not allowed to penetrate the membranes, the inner needle being kept retracted. Then the inner needle is projected to enter the theca and the stylet withdrawn. The flow of fluid is extremely slow; therefore a syringe should be attached to the butt end, and 5 c.c. of fluid withdrawn by suction. By this method, post lumbar-puncture headache can be abolished in practically all cases, and, should it occur at all, it will be extremely mild. (Allen I934: Erskine and Johnson 1938.) Bed in the recumbent position for 24 to 48 hours can be dispensed with and the patients can resume their usual avocations forthwith. Should the outer needle be pushed too far and enter the theca, the object of the Dattner needle is defeated, and symptoms are as likely to follow as with a needle of the ordinary type. The puncture is made in the mid-line in the interval between the 2 nd $\& 3$ rd or 3 rd \& $4^{\text {th }}$ lumbar spines, the inter-spinous interval being identified by pressure with the tip of the left thumb. The patient may be sitting up and bending forward, the easier method, or, if he is lying on the side, care must be taken to avoid any lateral flexion or rotation 
of the spine. Failure to enter the theca with these precautions and accurate technique indicates scoliosis, which must be discovered and allowed for. Local anæ: thesia should always be used.

In the prevention of congenital neuro-syphilis the same principles apply, bearing in mind that the treatment of early congenital syphilis means treatment of the fotus through the mother, starting as early as possible in pregnancy; in any case before pregnancy in an infected mother has advanced more than halfway. The usual methods of standard treatment are continued until the end of pregnancy, and a healthy child can be practically guaranteed. Pregnant women tolerate treatment well. Every woman in the child-bearing period of life who is known to have suffered from syphilis, congenital or acquired, or who has previously given birth to a syphilitic child or a syphilitic dead fotus, must be treated through pregnancy on the assumption that she is still syphilitic and capable of transmitting the disease to her offspring, even if her blood Wassermann is persistently negative, and she has had a full course of treatment herself. Any child born of a mother with a syphilitic history should have a blood Wassermann test as soon as possible after birth. A positive reaction at less than two weeks of age is not, however, proof of syphilis in the child, as it may be due to the transference of antibody from the mother. On the other hand, a negative blood Wassermann in the first few months may be followed by a positive reaction later, so that a further test should be made at one year old, before the child is definitely considered uninfected.

When a child is shown by clinical signs or serological tests to be suffering from syphilis, the cerebrospinal fluid should be examined and standard treatment begun. The fluid behaves as in adults. Positive changes are found in a considerable number, the majority of which become normal under standard treatment. If this does not occur, the treatment described later for congenital neuro-syphilis must be carried out. The necessity for this is well illustrated by the following case at present under the writer's care. A boy at the age of six was found to have Hutchinson's teeth and a positive blood Wassermann reaction. He was given standard treatment for 9 years, the blood remaining positive. At the age of I5 he was found to have Argyll-Robertson pupils and his cerebro-spinal fluid showed a positive Wassermann and other characteristic changes.

For details of the standard treatment of congenital syphilis the reader is referred to "Diseases of Children," Garrod, Batten, Thursfield \& Paterson, 3rd Edition, I934.

\section{B.-Treatment of Neuro-Syphilis.}

In meningo-vascular syphilis the drugs used in the routine treatment of somatic syphilis should be effective, as there is no barrier to their access to the diseased parts. In fact, if early syphilis is thoroughly treated with trivalent arsenobenzols (arsphenamines), and with bismuth, cerebro-spinal syphilis should not occur, except in those very occasional cases in which the defence reactions of the body fail. In one of the writer's cases the blood Wassermann was persistently negative despite the presence of a secondary rash. The rash cleared and treatment was continued; but during treatment a few months after the onset, an epileptiform fit occurred and positive changes were found in the cerebro-spinal fluid. Treatment of cerebro-spinal syphilis must be very cautious at first for fear of exacerbations. It is safest to begin with bismuth only, then add iodide, and finally arsenobenzol with low initial dosage. 
Chemo-therapy of parenchymatous neuro-syphilis presents a more difficult problem on account of the "blood-brain barrier." Substances with a large molecule cannot pass through the choroid plexus, or traverse the peri-vascular (Virchow-Robin), spaces that surround the blood vessels in the brain and spinal cord. Trivalent arsenicals (or the therapeutically active substances derived from them after injection) cannot pass. Bismuth, whether insoluble, lipo-soluble, or water-soluble forms are used, can only be recovered in minute quantities from the brain and cerebro-spinal fluid after intramuscular injection. The electrical charge carried by the metallic ion is said to be of importance in determining its penetration into the central nervous system, penetration being greater when this is negative (Hanzlik \& Spaulding, I93I). In some bismuth preparations, such as sodium bismuthate and sodium iodobismuthite, the bismuth ion is negatively charged, while in the official preparation bismuth salicylate it is positively charged. In the presence of meningeal and ependymal inflammation, however, the passage of trivalent arsenicals and of bismuth is probably facilitated somewhat.

Potassium and sodium iodide have been shown to penetrate into the cerebrospinal fluid when given in large doses by mouth and intravenously, and are claimed by various authorities to be of value in neuro-syphilis when given in doses of one drachm of the potassium salt by mouth three times per day, or in doses of one gramme increasing gradually up to ten grammes intravenously in ro per cent. solution, a course of 20 to 30 injections being given at intervals of one or two days. A test dose must first be given to discover idiosyncrasy. Iodides have no spirochæticidal action, but act by resolving exudates and inhibiting fibrosis. Therefore they are of value in meningeal forms of the disease.

Certain pentavalent arsphenamines and sulpharsphenamine have been found by Voegtlin, Smith, Dyer and Thompson, and also by Raiziss, to have a relatively high penetrating power into the central nervous system as compared with arsphenamine, neo-arsphenamine and silver-arsphenamine (Stokes I934). The pentavalent arsphenamines in most common use are Tryparsamide (sodium-n-phenylglycineamide-p-arsinate), Stovarsol (3-acetyl-amino-4-hydroxyphenyl-arsinic acid), and Acetyl-arsan (diethylamine-p-hydroxy-m-acetylamino-phenyl-arsenate). It must be borne in mind that these drugs, as exemplified by Tryparsamide, which is the only one that will be further discussed here, are feebly spirochæticidal, though Tryparsamide contains 24 per cent. of arsenic in organic combination. Therefore, if somatic syphilis co-exists with neuro-syphilis, bismuth therapy should be given at the same time. The dose of Tryparsamide is large, being I to 3 grammes dissolved in ro c.c. of sterile distilled water. The usual route is the intravenous one on account of the bulk of the injection, but this drug is not irritating to the tissues if given intramuscularly or subcutaneously. When reliance is placed on Tryparsamide in the treatment of latent or manifest neuro-syphilis, the treatment must be prolonged. At least 50 injections in repeated courses of 8-ro weekly doses must be given before obvious results can be expected. More than roo injections have been given to one case by some workers. Lorenz has given up to I,500 grammes. In addition to its specific action, Tryparsamide has a definitely tonic effect upon the patient. As regards toxic effects, nitritoid crises and unpleasant early reactions are practically unknown; jaundice and dermatitis occasionally, but rarely, occur. The one toxic effect to be looked for is amblyopia, which supervenes in 7 per cent. of patients. If this complication arises at all, it does so during the first Io to I5 injections-never thereafter. Any complaint of mistiness of vision or of narrowing of the visual fields necessitates immediate cessation of Tryparsamide treatment. In the majority the amblyopia clears up completely, some permanent impairment remaining in I per cent. to 3 per cent. of 
cases treated. In those that recover completely, Tryparsamide may be again cautiously administered. It is advisable before starting this form of treatment to have the ocular fundus and visual fields examined. Where there is syphilitic optic nerve degeneration of parenchymatous type, it is contra-indicated. Whenever there is contra-indication to Tryparsamide, sulpharsephenamine should be substituted, the best routes of administration of this drug being the intra-muscular or the deep sub-cutaneous in doses of 0.3 to 0.6 grammes dissolved in the minimum volume of sterile distilled water.

Mercury is beneficial in neurosyphilis (except in dementia paralytica), when given by inunction. Hoff was able to demonstrate mercury in the central nervous system of dogs eight hours after its inunction through the skin.

\section{Pyreto-Therapy.}

According to Engleman, fever facilitates in animals the accumulation of arsenic in the nervous system when neo-arsphenamine is injected during fever. Naturally no such experimental information is available in man. Even if this be true in man, fever has of itself a much more potent effect in the treatment of neurosyphilis, especially the parenchymatous forms, without the assistance of chemotherapy. Heat, usually in the form of hot baths, has been used since the fifteenth century in the treatment of syphilis which had then been introduced into Europe; this form of treatment has also been used extensively in Japan since the seventeenth century. The beneficial effects of induced pyrexia on the syphilitic lesions of rabbits was first studied in I9I9 and has been more extensively studied since I926. The first effective form of pyreto-therapy in neuro-syphilis was introduced by Wagner-Jauregg of Vienna in I9I7 in the shape of artificially induced malaria. More recently as the result of much research and experiment, mainly in the United States of America, pyreto-therapy by means of high-frequency currents, airconditioned cabinets and a combination of both electro-pyrexia and air-conditioning has been brought to a high degree of perfection.

Many other methods have been tried out, such as intravenous injections of T.A.B. vaccine and of Dmelcos vaccine (Ducrey's bacillus); intramuscular injections of sulphur in oil (Sulfosin), of a suspension of living saprophytic organisms (Saprovitan), of B.coli (Pyrifer), and of sterile milk. These are only mentioned to dismiss them as uncertain and unsatisfactory.

The mechanisms of pyreto-therapy in the cure of neuro-syphilis are still not completely agreed. The protagonists of mechanically produced fever maintain that fever alone is the only factor, acting as follows:-

I. Micro-organisms that have become completely parasitic and accustomed to the normal temperature of the host cannot flourish if the environment is radically changed.

2. Their growth is inhibited and they may be destroyed by an increase of $4.5^{\circ} \mathrm{F}$. maintained for hours. It is not necessary to reach the thermal death point of the organism, which, in the case of the treponema pallidum is I06.7 to $107.6^{\circ} \mathrm{F}$. maintained for six hours, or lower temperatures for longer periods (Bessemans' experiments in rabbits).

3. Natural bodily defence mechanisms are stimulated. Bessemans has also shown that treponemata in lymph nodes are more resistant to heat than treponemata in superficial lesions; thus the site as well as the nature of the organism must be taken into consideration. 
There are still those who believe that malarial infection has a specific effect in addition to its fever-producing effect. The arguments in favour of this are highly speculative, and will not be discussed here. Simpson has pointed out that the highest remission rate in malaria-treated dementia paralytica has been achieved in patients who had had at least fifty hours of fever above $102^{\circ} \mathrm{F}$.

Malaria Therapy. This is the method of pyreto-therapy in general use in Great Britain in the treatment of dementia paralytica, opportunity for using the American methods being still very limited. In the British mental hospitals malaria alone without chemo-therapy is usually relied upon. The benign tertian strain is most commonly employed, and the patient is infected by means of mosquito bites or by injection of $2 \mathrm{c} . \mathrm{cm}$ of blood from another infected patient subcutaneously, intramuscularly or intravenously. The incubation period varies from 9 to 16 days, being shorter in the patients injected intravenously. Before the fever is thoroughly established frequently minor rises of temperature are apparent for a day or two. The fever produced is either tertian or quotidian, and frequently starts as the former, becoming the latter as the disease progresses. During the bouts of fever, the temperature must be taken every I5 minutes and prevented from rising above I0 $5^{\circ} \mathrm{F}$. by tepid sponging, if necessary. If the condition of the patient permits, I2-I4 bouts of pyrexia are allowed before quinine is given to terminate the attack, in doses of $5 \mathrm{gr}$. of the bi-hydrochloride daily for I5 days. If the patient's condition demands, temporary interruption of the fever for Io-20 days may be achieved by giving one dose of $5 \mathrm{gr}$. The treatment is severe and must not be lightly undertaken. A thorough clinical examination must be made to exclude tuberculosis, diabetes, severe anæmia, rapidly advancing neuro-syphilis, renal and cardio-vascular disease, though valvular disease is not an absolute contra-indication if the myocardium is satisfactory. Septic foci should be first eliminated. Obesity calls for special care. The indications for terminating the malarial attack are persistent vomiting, cyanosis, collapse during the paroxysm, seizures, restlessness, tachycardia and early jaundice, and stained blood films showing more than 35 parasites in 25 fields, using a one-twelfth oil immersion lens and a No. 2 eyepiece (Nicol). It is also a danger signal if the temperature does not fall to normal between the paroxysms.

In patients who are resistant to infection by one strain of malaria parasite, either from previous therapeutic malaria, or from having previously acquired the disease naturally, different strains of benign tertian malaria may be used, or recourse may be had to infection by malignant sub-tertian parasites, a procedure that has been successfully and safely carried out in many cases by James, Nicol and Shute. The quartan strain is useful, not only in patients resistant to benign tertian, but also in debilitated patients, as the resulting illness is less severe and may be allowed to run for 20 peaks of fever. If necessary, the fever may be stopped for two weeks by 5 grains of quinine. Where there is difficulty in inducing malaria despite the use of different strains, success may sometimes be achieved by giving protein shock during the incubation period, intravenous T.A.B. vaccine being used for the purpose in one or two doses of 25 millions and 50 millions respectively. Another method of provocation is to infect with both benign tertian and quartan malaria at the same time. Even after apparent failure occasionally the malarial attack supervenes after an abnormally long incubation period, the longest in the writer's experience being 2-3 months in a patient highly resistant to malaria.

Malarial relapses occur in 60 per cent to 70 per cent. of cases infected by mosquito bites with benign tertian parasites, usually 6-7 months after the primary 
attack has been stopped by quinine. These relapses are milder than the primary attack and can generally be left to subside spontaneously. Cases infected with benign tertian by blood injection do not relapse. In malignant tertian infection recrudescences of mild type at short intervals after the primary infection are common irrespective of the method of infection employed. This parasite is resistant to quinine in 80 per cent. of cases, but responds well to atebrin. Quinine administered in the incubation period of any type of malaria fails to prevent its development; but plasmoquine is an effective prophylactic should the patient develop an intercurrent disease after infection rendering a malarial attack undesirable.

Electro-pyrexia was first introduced therapeutically by Neymann and Osborne in 1927. The methods in present use are:-

1. Inductothermy (electro-magnetic induction by ultra-short waves), the patient being enclosed in an insulating bag up to the neck, and the electrodes being either passed twice round the outside of the bag or coiled upon it.

2. The radiothermy cabinet in which use is made of both heating by ultra-short waves and by air conditioning.

For details the reader is referred to "Artificial Fever," Neymann 1938. In brief, the technique advocated is 20 bi-weekly treatments, the temperature being maintained above $103.5^{\circ} \mathrm{F}$. for eight hours at each treatment, and for two of the eight hours at over $105.8^{\circ} \mathrm{F}$. It is recommended that this treatment be combined with and followed by Tryparsamide therapy.

The Kettering Hypertherm was introduced by Simpson, Kettering and Sittler in 1933, when they discovered that pyreto-therapy could be carried out equally well in an air-conditioned cabinet without the aid of high frequency currents.

The course advised by Simpson is 10 (occasionally more) weekly treatments of five hours at $105^{\circ} \mathrm{F}$. to $106^{\circ} \mathrm{F}$-minimum 50 hours of fever sustained at this level. During the ten weeks the fever treatment is combined with 30 injections of $0.3 \mathrm{grm}$. of neoarsphenamin and 30 injections of a bismuth preparation $(0.2 \mathrm{grm}$. of metallic bismuth per dose), or with a course of injections of Bismarsen (bismuth arsphenamine sulphonate). A dose of the anti-spirochætal drug is given half an hour before each fever treatment. Subsequently a follow-up course of 20 injections of the selected chemical agent is given at weekly intervals.

The above-mentioned forms of pyreto-therapy can only be carried out by a specially trained team as there are technical details of extreme importance if safety is to be assured and the risks of collapse, burns, dehydration, loss of salt and hyper-pyrexia are to be avoided (sweat loss is $\frac{1}{2}-\mathrm{r}$ litre per hour). Heat stroke occurs at $108.5^{\circ} \mathrm{F}$. In skilled hands the risk to life is considerably less than with malaria therapy. The contra-indications are:-

I. Decompensated heart disease.

2. Advanced organic brain disease, especially with cerebral arteriosclerosis.

3. Nephritis.

4. Tremors and convulsions (risk of hyperpyrexia).

5. Cutaneous anæsthesia (risk of burns).

6. Senility and cachexia. 
Other methods of overcoming the blood-brain barrier:-

1. Spinal drainage after intravenous injection of arsphenamine-a procedure of doubtful value.

2. The direct injection of the drug into the sub-arachnoid space (Ravaut: Gennerich) dissolved in normal saline, distilled water, or cerebro-spinal fluid. Owing to the irritant nature of the injected solution, this method is risky, and only minute doses can be given; e.g., $0.5 \mathrm{mg}$. of neoarsphenamine. It has fallen into disuse, though said to be of value in the treatment of certain tabetic symptoms. Since the recent introduction of Mapharside, concerning which it is claimed that the same therapeutic effect can be obtained as with a dose of neoarsphenamine ten times larger (confirmed clinically in the treatment of somatic syphilis), it seems likely that endo-lumbar injection could be carried out with greater safety using Mapharside.

3. The endo-lumbar and intra-cisternal injection of salvarsanised serum (Swift \& Ellis). After an intravenous injection of neoarsphenamine, blood is withdrawn 40 minutes later, and the serum diluted with an equal quantity of saline is injected intrathecally or intracisternally. For intracisternal therapy, 40 c.cs of blood are taken, yielding approximately $20 \mathrm{c} . \mathrm{cs}$ of serum. This is heated to $55^{\circ} \mathrm{F}$. for one hour and kept in an ice chest till next day, when it is diluted, warmed to blood heat, and injected by gravity into the cisterna magna after the removal of 45 c.cs of cerebrospinal fluid. The usual course consists of six treatments given weekly or fortnightly. A further course may be given later if desired. (For the technique see " Neurological Effects of Syphilis," Oxford University Press, 1933, p. 63.) The actual amount of arsenic injected into the subarachnoid space in this way must be small. One hour after intravenous injection only 10 per cent. of the arsenic can be identified in the blood, and none after two hours; 60 per cent. disappears from the blood stream within a few minutes. Therefore Ogilvie added additional neoarsphenamine to the "salvarsanised" serum at the rate of 0.1 to $0.25 \mathrm{mg}$. per dose. The suggestion has been made that " salvarsanised" syphilitic serum contains antibodies which combat the infection in the nervous system.

\section{Prognosis in treated Neuro-Syphilis.}

Without the use of pentavalent arsphenamine or pyreto-therapy, the chance of cure by standard anti-syphilitic treatment depends directly on the ratio of inflammatory to degenerative processes. Thus the order of diminishing response is:-

Meningeal syphilis.

Cerebro-spinal syphilis.

Tabes.

Vascular disease.

Dementia paralytica.

Optic atrophy (parenchymatous) - no benefit at all.

Tabetic lightning pains are benefited in over 50 per cent., and bismuth injections relieve, at least temporarily, subjective tabetic symptoms. Tabetic crises are benefited in over 25 per cent.

Arsenobenzol injections, especially at the beginning of treatment, may temporarily aggravate symptoms (Herxheimer's therapeutic shock). Specific drug neurotropism is rare; e.g., arsenical neuritis; but it must be taken into account if there is parenchymatous optic atrophy. 
The order of reversal of cerebro-spinal fluid changes under any form of treatment is:-

Cell count.

Protein.

Colloidal precipitation curve.

Wassermann and flocculation reactions, which may take 4-5 years, if reversal occurs at all.

Clinical and serological improvement do not run parallel; thus clinical recovery or remission may be seen without serological cure, and occasionally active neurosyphilis is encountered with a normal cerebro-spinal fluid, and possibly also with a negative blood Wassermann. Obviously the persistence of cerebro-spinal fluid abnormality is an indication for continuing treatment.

Results of Tryparsamide therapy. This drug is of value in all forms of neurosyphilis including early dementia paralytica, and its administration should not be abandoned unless no benefit has resulted from 70-100 injections.

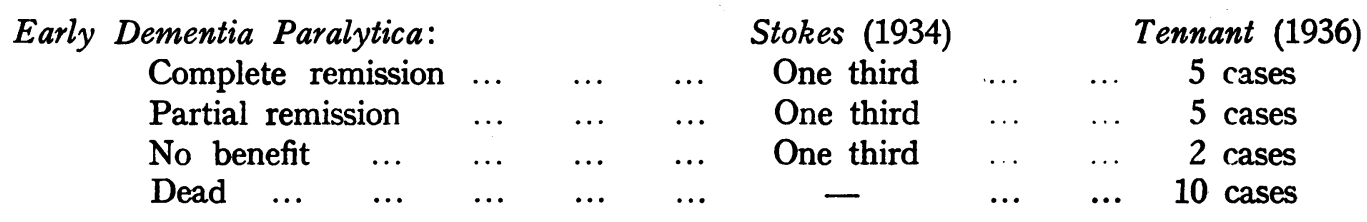

Other workers have given varying percentages of complete clinical remission in small series of cases varying from 39 per cent. to 80 per cent. Reversal of the cerebro-spinal fluid to normal may be expected eventually in about one third of the cases. The writer obtained complete remission with tryparsamide treatment, for a period of Io years, till his death from pneumonia, in a professional man who developed dementia paralytica after one year of standard treatment for a syphilitic aortic regurgitation.

In tabes the results of any treatment are difficult to assess, as this disease may undergo spontaneous arrest without treatment and remain static for many years; in other cases there may be progressive deterioration despite energetic treatment of all kinds. The state of the cerebro-spinal fluid is no index of the clinical picture. Tryparsamide has been favourably reported on by Moore \& Sutton (I926), Lees (I932) and others. Keith \& Lemarquand (I929) reported marked improvement in 7 , and slight improvement in II of a total of 24 cases, the pains and ataxia being particularly ameliorated in those responding, as well as the general health. Neymann (1938) reports less optimistic results, pains and crises being relieved in only a small proportion. Cormia (I934) states that tryparsamide is less effective in tabes than in other forms of neuro-syphilis despite the higher proportion of serologic reversals. In many tabetics it is contra-indicated on account of parenchymatous optic atrophy. Nevertheless Cady (I930) reported I2 per cent. improved and 50 per cent. arrested in I6 cases of primary syphilitic optic atrophy, and 60 per cent. improved or arrested in Io cases with contracted fields. Lees (I932) secured complete arrest in 50 per cent. of 2I cases of syphilitic optic atrophy.

The Results of Malaria Therapy. In dementia paralytica the benefit to be expected varies with the promptitude with which the diagnosis is made and treatment begun. The statistics from mental hospitals, whence the most numerous 
cases have been reported, are disappointing, because a certified case is a late case except when the onset of mental symptoms is abrupt. The manic and grandiose types respond far better than the depressed, paranoid, stuporose and simple dementing types, partly because earlier diagnosis is likely. Stoddart has pointed out that the recovery rate after malaria drops from 60 per cent. to 8 per cent. when the disease remains untreated for more than I8 months from the onset.

In a survey of 3,079 cases made by Kraepelin there was full remission in 2I $\frac{1}{2}$ per cent., improvement in $2 I_{\frac{1}{2}}$ per cent., and no improvement in 57 per cent., with a death rate of Io per cent. or more. Stokes reports full remission in 39.I per cent., partial remission in $2 \mathrm{I} .8$ per cent., and death in IO-I2 per cent., alleging that malaria alone gives slightly better results than tryparsamide alone.

\begin{tabular}{cccccccc} 
Nicol \& Hutton & $(1936)$ & & Discharged. & \multicolumn{2}{c}{ Not discharged. } & & Dead. \\
50 women & & $\ldots$ & 22 (relapsed 4) & $\ldots$ & 18 & $\ldots$ & 10 \\
84 men & $\ldots$ & $\ldots$ & 25 (relapsed 2) & $\ldots$ & 33 & $\ldots$ & 26
\end{tabular}

Tennant (1926) - 62 cases selected as favourable and treated with malaria and tryparsamide-Good remission 27, partial remission 11, mental hospital 8, and dead 20 (including 4 in full remission).

Nicole \& Harrison (1936) Discharged. 5-year cures. 10-year cures 103 malarialised cases 30 per cent. ... 18 per cent. ... 14 per cent.

These cases were observed for 10 years or more, 34 having second attacks and 5 third attacks, and 17 being given arsenic as well, of which 4 gave 10 -year cure.

The effect of malaria therapy on the cerebro-spinal fluid:-

Nicol \& Hutton, 1936.

Completely negative.

$\begin{array}{ccc}\text { Reduced. } & & \text { Strong+ } \\ 10 & \ldots & 4 \\ 22 & \cdots & 15\end{array}$

The majority of the men were observed for not more than one year.

Nicole \& Harrison:-

(a) Wassermann $\quad \ldots \quad$ After 4 years over 50 per cent. negative.

10-14 years only 2 of 35 positive.

(b) Cell count $\ldots \quad \ldots \quad$ In 1 st year over 50 per cent. normal.

Only 1 abnormal of 35 after 9 years.

(c) Globulin $\quad \ldots \quad \ldots \quad$ After 6 years over 50 per cent. normal.

(d) Total protein $\quad \ldots \quad$ None abnormal after 9 years.

(e) Lange curve $\quad \ldots \quad$ After 8-14 years over 50 per cent. normal.

In 3rd year paretic curve reduced to normal in over 50 per cent.; only one of 75 remained paretic in type after 7 years.

The proportion of normal fluids increased with elapse of time, but the clinical results and the findings in the fluid bore no relationship to one another.

The cerebro-spinal fluid findings and histopathological findings after malaria therapy are in agreement. Nicol and Hutton found that in patients dying with positive serology, the typical histological appearances of dementia paralytica were found in their brains, and vice versa. The death rate from malaria therapy 
averages about Io per cent. These workers claim that malaria is an active spirochæticidal agent in 80 per cent. to 85 per cent. of neuro-syphilitics, as judged by the proportion in which a normal fluid is produced by one course, while in the remainder a second course will achieve this in most, if not in all. Despite this, the cures in dementia paralytica amount to only 20 per cent. owing to irreparable damage before treatment is started, which may progress despite cure of the brain infection. In about one-third of the cases treated the disease is arrested and life prolonged, often for many years, creating the problem of an increased mental hospital population.

If the patient does not respond by mental improvement after the first adequate malarial course, he will never do so, and a further course is contra-indicated. In no case does immediate improvement show itself, but improvement should be apparent in 6 months, and if marked improvement has not taken place in 2 years, none will ever occur. A second course $\mathrm{I}-2$ years after the first is indicated where good remission is obtained from the first, but where clinical or serological evidence of dementia paralytica persists, the state of the fluid being checked by repeated lumbar puncture till completely negative. In successful cases it would appear that 2-4 years is required for the fluid to become normal; if this does not occur, relapse is likely. Further malaria will improve some of the clinically relapsed cases, but should be used to anticipate this event. The blood Wassermann and flocculation tests are no guide, and usually remain positive. Unfortunately some of the serologically successful cases remain with-

(a) Permanent non-progressive deterioration;

(b) Progressive physical and mental deterioration with slight or absent histopathological evidence of the disease post-mortem;

or (c) With a non-syphilitic psychosis that happens to be coincidental.

The real answer to the problem, and the only hope of normal useful life in the majority, is the treatment by tryparsamide and/or pyretotherapy of latent neuro-syphilis, and the diagnosis and treatment of clinical neuro-syphilis at its inception. O'Leary's results of malaria therapy in asymptomatic, persistently sero-positive, neuro-syphilis were-

Reversal of cerebro-spinal fluid abnormality

35 per cent.

Improvement

(Observation $I-6 \frac{1}{2}$ years).

The fate of the untreated paralytic should be contrasted with the abovementioned results of therapy. Nicole and Harrison state (I936), that of 45 untreated cases $4 \mathrm{I}$ were dead in 2 years and all in 7 years. None were discharged from the mental hospital. The usual life expectation is $2-5$ years with an average of 3 years, cases with frequent convulsions usually dying in about 6 months. Fulminating cases are occasionally seen to terminate fatally in from one week to a few weeks. Female cases, congenital dementia paralytica and tabo-paresis tend to run a slow course. As regards spontaneous remissions, the usually accepted figure is under 4 per cent., though a considerable variation exists in the figures given by various authorities. A spontaneous remission may last some months, but not often as long as 2 years. The most favourable in this respect is the purely manic type, in which survival up to Io years has been observed.

In Tabes, as always, the effect of malaria treatment is difficult to evaluate. Improvement has been reported in 35 per cent. to 75 per cent. by various authors. 
Yates (I934), compared the results in 32 malarialised tabetics with the results obtained by standard treatment in 25 tabetics. A good result was obtained in 34 per cent. of the former and in 54 per cent. of the latter, there being no response to either form of treatment in the remainder. The most interesting points in his report were that cases of more recent onset, (5 years or less), in both series responded the best, and that the most favourable results were obtained when there was evidence of meningeal involvement in the shape of a cell count of 30 per c.mm. or more and a protein percentage of $80 \mathrm{mg}$. per IOO $\mathrm{c} . \mathrm{cm}$. or over in the cerebrospinal fluid. Six cases fulfilling these conditions all did well. One of the writer's cases bears out Yates' views: the onset was acute with loss of the use of the legs: for several years now he has been back at work as a window cleaner, but still has lightning pains. It is clear from the foregoing that malaria in tabes does not promise any more definite and indisputable benefit than can be obtained by standard treatment. The main indication would appear to be intractable crises and lightning pains, sufficiently severe to justify the risk of giving this treatment, seeing that tabes itself is a relatively non-fatal disease. Even when relief is obtained by malaria, it may be only temporary.

The Results of Electro-Pyrexia :- Neymann reports complete remission in 27 per cent., improvement in 26 per cent. and death from treatment in 2 per cent. of cases of dementia paralytica. The prognosis in terms of the type of psychosis is the same as in malaria therapy. The serological results show improvement in the usual order, but are not parallel with the clinical results. He claims that electro-pyrexia gives better results than malaria with a negligible death rate, and that the results are better still if this treatment is combined with and followed by Tryparsamide. It is difficult, of course, to compare the results obtained by various workers, when the diagnostic criteria and the stage at which treatment is begun are not necessarily comparable.

In tabes he reports improvement in 66 per cent., no improvement in 34 per cent., disappearance of pains being a feature in successful cases, and crises being sometimes terminated. In optic atrophy the results have been so far inconclusive.

The Results obtained by the Kettering Hypertherm:-Simpson, by the method already described; gives the results summarised below:

Dementia Paralytica. Remission. Improved. Not improved 27 cases 21 5 1 (demented).

C.S.F. (after several months)_Cells; remained normal or reduced to normal in all.

Protein; remained normal in 4, reduced to normal in 15 , increased in 5, and unchanged in 3.

Colloidal gold curve; normal in 2 , reduced in 19 , increased in 5 , unchanged in 1.

Kahn test; negative in 6 , less positive in 15 , unchanged in 6.

Tabes 14 cases: The chance of improvement was inversely proportional to duration. Root pains were abolished in all, but recurred in 3.

$\begin{array}{lcccc}\text { Ataxia } & \text { Normal gait. } & 75 \% \text { improvement. } & 50 \% \text { improvement. } & \text { No benefit. } \\ 11 \text { cases } & 2 & 5 & 2\end{array}$

In the 2 cases recovering normal gait, the duration was 3 months and 2 years respectively. 
C.S.F.-Kahn test became negative in 4, remained negative in 1 , became less positive in 3 , and was unimproved in 6 .

Tabo-paresis: -8 cases were all improved except 2 demented patients.

Asymptomatic neuro-syphilis:-the Kahn reactions in the cerebro-spinal fluid became negative in all.

Optic neuritis and neuro-retinitis:-14 cases, in all of which the disease was arrested.

Optic atrophy:-No change in any, but possibly arrest. This condition did not contra-indicate fever therapy.

No deaths occurred as the result of this form of pyreto-therapy.

The Results of intracisternal salvarsanised serum :-This method is now rarely used. Purves-Stewart in England advocated it as an addition to malaria treatment in dementia paralytica. His published results are, however, few in number, being confined to 29 private cases treated from I9I9 to I928. Of these, 22 cases were treated by both malaria and the Swift-Ellis methods, and II gave full clinical remission, 2 were improved, 6 were unimproved, 2 died and I was lost sight of unimproved soon after treatment terminated. Of those in full remission, 8 showed serological cure and 2 serological improvement; of those clinically improved, I showed serological improvement and the other none; of the 6 clinically unimproved, 3 showed serological improvement and 2 none (I not reported on). The SwiftEllis technique has been reported on favourably by Moore in the treatment of syphilitic optic atrophy, provided that the acuity of vision in the better eye has not fallen below one-third of normal. It has also been advocated in basilar moningitis.

Endo-lumbar salvarsanised serum:-The writer has no experience of this mcthod, but it was formerly used extensively in other countries.

Stokes summarises the indications as being:-

1. Moderate resistance, especially of the meningeal phase of early meningo-vascular syphilis.

2. Low tabes with bladder and sexual disturbances and marked lightning pains and ataxia.

It is contra-indicated in:-

1. Rapidly progressing cord involvement.

2. Acute cerebral processes with signs of increased intracranial pressure.

3. Gastric crises, severe attacks of which may be precipitated.

\section{Summary.}

In placing any case in a particular group the most prominent features are taken into account. Involvement of the nervous system is always initially meningeal, and the clinical types are not always clear cut, especially at first.

1. Cerebro-spinal syphilis:-

(a) Standard treatment with bismuth, iodide by mouth and arsenobenzol.

(b) Supplementary treatment with Tryparsamide.

(c) In some cases large doses of sodium iodide intravenously. 
(d) Pyreto-therapy indicated only in cases resistant to the more usual forms of treatment. When benefit results it is probably due to vaso-dilatation.

(e) Endo-lumbar salvarsanised serum in resistant meningeal forms.

2. Dementia paralytica:-

(a) Tryparsamide as soon as diagnosed together with bismuth.

(b) Pyreto-therapy as soon as possible in patients fit to undergo it, followed by Tryparsamide and bismuth.

(c) Further pyreto-therapy if necessary, and if indicated 1-2 years after the first course.

(d) Intracisternal salvarsanised serum might be justified in occasional cases, but its value is not definitely determined.

3. Tabes:-

(a) Remove all foci of infection.

(b) Course of neo-silver-arsphenamine followed by course of bismuth.

(c) Potassium iodide by mouth, or sodium iodide intravenously.

(d) Tryparsamide.

(e) Pyreto-therapy may be tried in selected cases when other forms of treatment fail to relieve intractible pains and crises.

(f) Endo-lumbar salvarsanised serum according to indications mentioned above.

4. Primary Syphilitic Optic Atrophy:-Atrophy cannot regenerate, but if there is active neuritis, the indications are for iodides, the cautious trial of tryparsamide, and perhaps intracisternal salvarsanised serum. Pyreto-therapy as a last resort will, at least, do no harm. Standard treatment by arsphenamine is of no avail, and H. Lauber (1938), regards it as dangerous in so far as it lowers blood pressure without lowering intra-ocular tension. He suggests that the lowering of the latter by continuous instillation of 2 per cent. pilocarpine into the eye or by cyclodialysis is indicated.

\section{Adjuvant Treatment of some Tabetic Symptoms:-}

(a) Lightning pains-General hygiene, rest and warm climate.

Symptomatic relief with pyramidon, aceto-salicylic acid and sodium bromide.

Abortive treatment sometimes succeeds with adrenalin $1 / 1,000$, $\mathrm{m} \times$, subcutaneously.

(b) Ataxia-Re-educational exercises.

(c) Gastric crises-Symptomatic relief with chloral and bromide, or with Spasmalgin (Roche).

Abortive treatment with adrenalin injection.

It is not possible to set any limit to the period of treatment and observation required in neuro-syphilis.

The Treatment and Prognosis of Congenital Neuro-Syphilis.

The serologically or clinically syphilitic infant with an abnormal cerebro-spinal fluid at the outset should be given standard treatment with arsphenamine injections 
and mercury by mouth, followed by bismuth injections, if necessary, the dosage being adjusted according to body weight. In small children it is easier, and therefore usual, to give arsenic intramuscularly in the form of sulfarsenol or some similar preparation. The superior longitudinal sinus, which is a useful source from which to obtain blood for test before the anterior fontanelle is closed, should never be used as a portal for arsphenamine injections. An infant, breast fed by a mother herself receiving arsenical treatment, obtains some arsenic in the milk, so that its injected dose should be reduced somewhat to allow for this. Stovarsol (Orarsan, Spirocid), by mouth instead of arsenical injections is apparently effective and largely used in Germany.

If the cerebro-spinai fluid remains abnormal after one year of standard treatment, a change should be made to tryparsamide and bismuth. Both can be given intramuscularly mixed in the same syringe, as suggested by Nabarro, who recommends a dosage of $0.5 \mathrm{grm}$. of tryparsamide at age $2-3$ years, and up to I.5-2 grm. per injection for older children: Bisoxyl (5 per cent. bismuth oxychloride in 5 per cent. glucose solution) is mixed with it, an infant weighing 4 kilos receiving $0 . \mathrm{I}$ c.cm. initially, which is increased by $0 . \mathrm{I}$ or $0.2 \mathrm{c.cm}$. per injection up to I c.cm. at the tenth injection of the first course. This régime is continued for $I-2$ years. If the fluid is then still abnormal, malaria or the Swift-Ellis method is resorted to.

In clinical neuro-syphilis the same lines of treatment are followed. In older children with meningo- vascular syphilis neo-silver-salvarsan intravenously has been found useful and intravenous sodium iodide has been tried.

The results of treatment are much the same as in the corresponding adult types, except that the prognosis in juvenile dementia paralytica is definitely worse, especially in the young. The most optimistic results are those recorded by Lees, (I93I), who reported 2I.4 per cent. of clinical recovery in I4 patients, with a mortality of I 4.3 per cent. after malaria, tryparsamide, bismuth and potassium iodide. Potter, (I933), obtained complete remission in 5 of 38 cases with malaria and tryparsamide, noting that the prognosis was better in patients who were normal before the onset of dementia, in whom the onset was after adolescence, and in whom the symptoms were of not more than 2 years duration, and were of the euphoric or confused type. Neyman, (1938), found records of 67 malariatreated cases of which 8 were markedly improved, 20 improved, and 39 unimproved, there being no complete remissions. In the 9 cases on record treated by electro-pyrexia no complete remissions had been obtained. It seems that syphilitic disease of the developing brain is bound to result in permanent impairment despite the most effective therapeutic methods known. The only hope is in cases of recent onset after puberty. 